\title{
Research on Flight Grasps System of Four-rotor Aircraft Based on Vision
}

\author{
Zhennan $\mathrm{MA}^{1, \mathrm{a}}$, Feng $\mathrm{Yu}^{2, \mathrm{~b}}$, Xiaoming Feng ${ }^{2, \mathrm{c}}$ and Bingbing $\mathrm{Yan}^{2, \mathrm{~d}}$ \\ School of Mechanical Engineering, Jiamusi University, Jiamusi154007, China \\ amazhennan-12345678@qq.com, ${ }^{b} 779222180 @ q q . c o m$, \\ c1159527506@qq.com, dyanbingbing@126.com
}

Corresponding author: Bingbing Yan

Keywords: OpenCV, Camshift, Four-rotor.

\begin{abstract}
In order to ensure interior target is grabbed by the four-rotor aircraft, the camsift algorithm of OpenCV is adopted, through the recognition of the target, at the same time, the positioning of the aircraft and alignment of target are conducted to achieve grab. The target object is placed on the ground and the image is collected, the center point of the target object and relative position of the aircraft is determined, the gesture of aircraft is adjusted by airborne vision sensor, until the target is located below the aircraft. When the aircraft dropped to the height of the calculation, the images are captured by vision sensor of end-effector, until gripper with two degree of freedom is aligned with the object be grabbed. The results show that the scheme can meet the requirements of flight grasps of the four-rotor aircraft, and with high precision.
\end{abstract}

\section{Introduction}

The key to the flight grasps of aircraft is the positioning in indoor of four-rotor and the positioning of the gripper for target ${ }^{[1]}$, the inertial unit normally used as the primary sensing device of measurement ${ }^{[2]}$ in four-rotor aircraft ${ }^{[3]}$, but only the requirements of flight stability is satisfied, the positioning of the aircraft can't be carried out ${ }^{[4]}$. The GPS positioning system is limited in the indoor experiments, so a visual sensor onboard the aircraft is introduced of to achieve the positioning of the aircraft. Indoor fixed flight aircraft due to an error, a scheme that end-effector with two degree of freedom based on vision of the target is introduced to locate the target and improve the grasp accuracy.

\section{The Four-rotor Aircraft with End-effector}

Four-rotor aircraft is controlled by PC machine, as a result of the gesture control unit, which makes the control of the four-rotor is relatively simple, the aircraft is connected with the PC machine via cable, the image captured can be recorded in real time, and has the effect of manipulating the first perspective. Aircraft has two cameras, the first camera as down-looking camera with resolution of $640 * 480$, the sampling frequency of 15 frames / sec, the second camera as the end-effector camera with resolution of $640 * 480$, the sampling frequency of 15 frames / sec.

The four-rotor aircraft has self-stabilized mode, aircraft control commands can be sent to control pitch, roll, yaw and up, down ${ }^{[5,6]}$ and other actions of aircraft to achieve the positioning of the aircraft based on acquired image information of down-looking camera. Grab parts and mechanical hand are connected to two servo platform, which makes the mechanical hand with the $\mathrm{Z}$ axis and the $\mathrm{X}$ axis rotation of two degrees of freedom, and based on acquired image information of end-effector camera, the gripper relative to the gesture of the target is obtained to control the servo motion.

\section{Grasp Scheme of Four-rotor Aircraft}

The down-looking camera of aircraft with low resolution and high frame rate, which fast processing and update of the image can be realized. The positioning of the aircraft is achieved by the 
movement and tracking algorithm in OpenCV , the implementation steps of specific programs are as follows:

1) the target object is determined: an object with fixed color (blue) is placed on the indoor ground, the size of the object (diameter $32 \mathrm{~mm}$, height $15 \mathrm{~mm}$ ) in the holder clamping range.

2) the position of the aircraft is determined: When the object of the ground is captured by the down-looking camera, motion tracking is conducted according to the color of the object, the upper left corner of the image as pixel coordinate origin "O", which the pixel coordinates of the center of the target are determined, the control commands are sent to arduino controller by PC machine to control aircraft, the target is located in the center area of the picture, at the same time, the aircraft directly above the target.

3) the height of the gripper is determined: the reasonable height of gripper is calculated by the size of the object, the position of servo platform and down-looking camera, steering angle, the clamping mechanism.

4) grasp: when the aircraft's position is determined, the PC machine start to adjust the angle of the two servos by analyzing the image of the target that captured by the end-effector camera.

\section{Positioning Algorithm of Four-rotor Aircraft}

Positioning Algorithm of $\mathbf{X}, \mathbf{Y}$ Plane. The target object with color fixed on the ground as reference for the positioning algorithm of four-rotor aircraft. The pixel coordinates of the image is obtained to control the position of aircraft. The movement and tracking of target object is achieved by the camshift algorithm in OpenCV vision library. The image is converted to color probability distribution from the color histogram model, the size and location of a search window is initialized, and the location and size of the search window is adjusted adaptively according to the result of the last frame, the center position of the current image is located. The flow chart of algorithm is shown in Fig.1. the camshift algorithm is used to track the target object and plot the tracking area. The image is collected by the down-looking camera is shown in Fig. 2 .

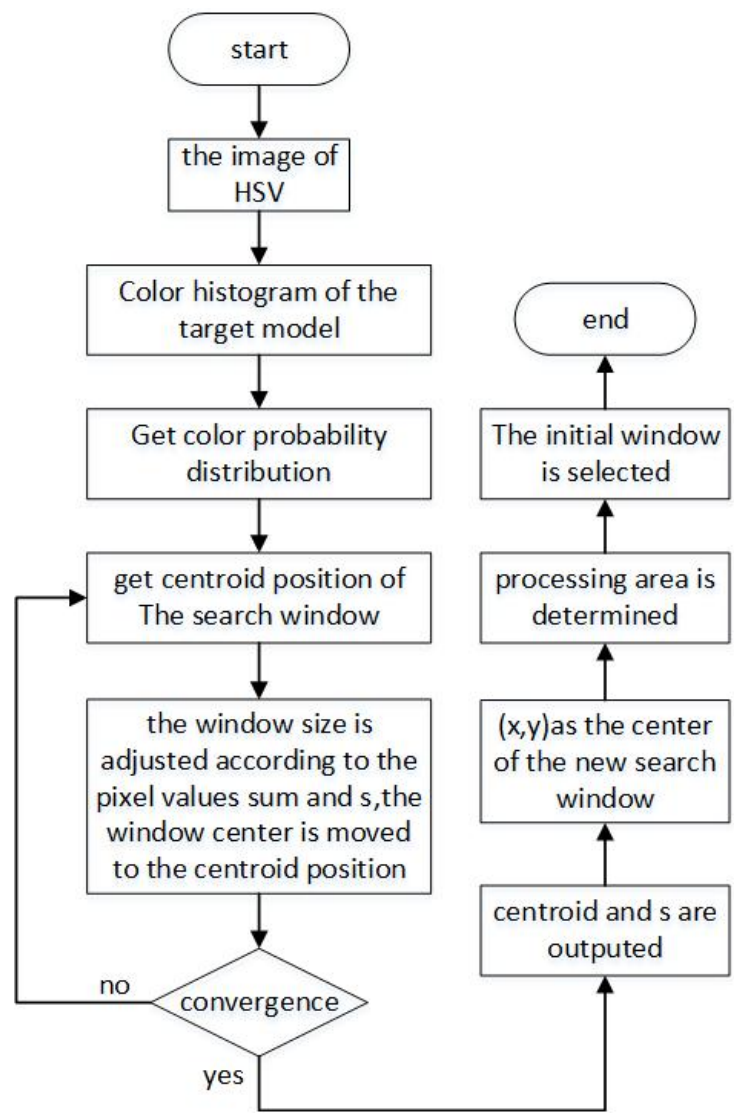

Fig. 1 The flow chart of camshift algorithm

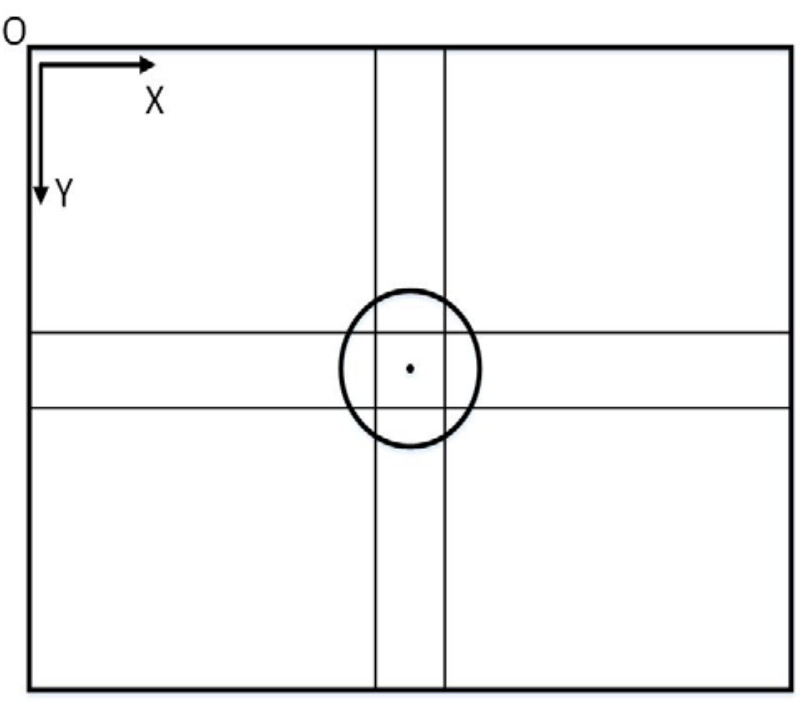

Fig. 2 The positioning map of four-rotor aircraft 
Height Positioning Algorithm for Gripper. When the target object is captured by the down-looking camera of aircraft, after the positioning of X, Y plane is completed, it will hover above the target about $1 \mathrm{~m}$. At this time, the camshift algorithm is used for end-effector camera to track the target object, the servo platform is controlled by the command of PC machine, which enables the target object is aligned with the gripper. The structure of the gripper and servo platform are shown in Fig. 3. The aircraft can accurately grasp the object, the height range of the aircraft is calculated according to the size of target object. Formula (1) is as follows.
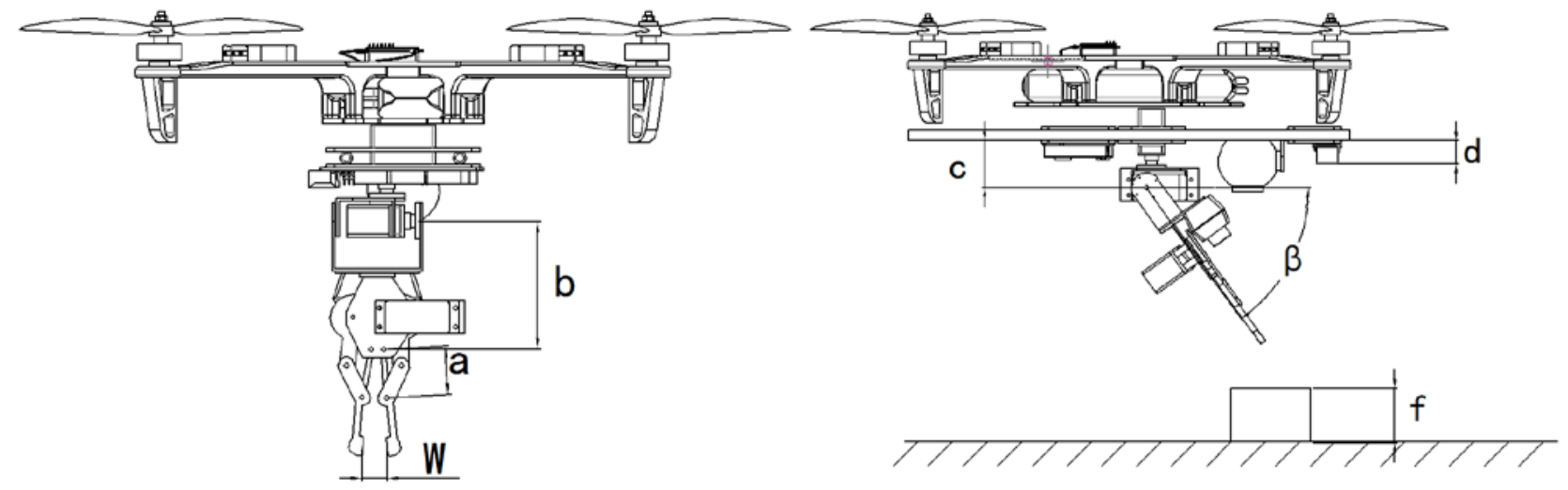

Fig. 3 The structure of the gripper and servo platform

$$
z_{g}=z_{p}+d-e-\sin \beta\left[\sqrt{a^{2}-(w-5)^{2}}+b+30\right]
$$

a- the length of connecting rod of gripper.

b- the distance from gripper connecting rod to the second servo shaft.

c- the height from the airframe to the second servo shaft.

$\mathrm{d}$ - the height from the airframe to the ultrasonic module.

$\beta$ - the rotation angle of the second servo.

$\mathrm{z}_{\mathrm{g}}$ - the height of gripper distant the ground.

$\mathrm{z}_{\mathrm{p}}$ - the height of aircraft.

$\mathrm{w}$ - the width of target object.

Among $z_{p}$ is measured by ultrasonic sensor in real time. It is known that the height of the target object is $\mathrm{f}$, when $\mathrm{z}_{\mathrm{g}}<\mathrm{f}$, the object can be grabbed by gripper.

\section{Experiment}

The gesture that pitch, roll, yaw and up of four-rotor aircraft is controlled according to the centroid coordinates of object and position deviation of center point of image, which enables the target object is located in the center of image. The positioning images of aircraft are as follows:

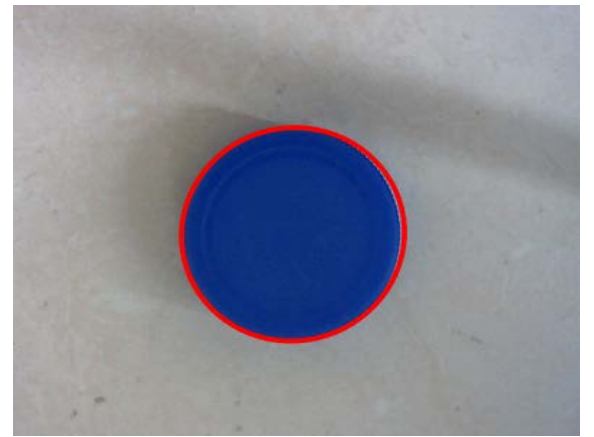

Fig. 4 The positioning images of aircraft

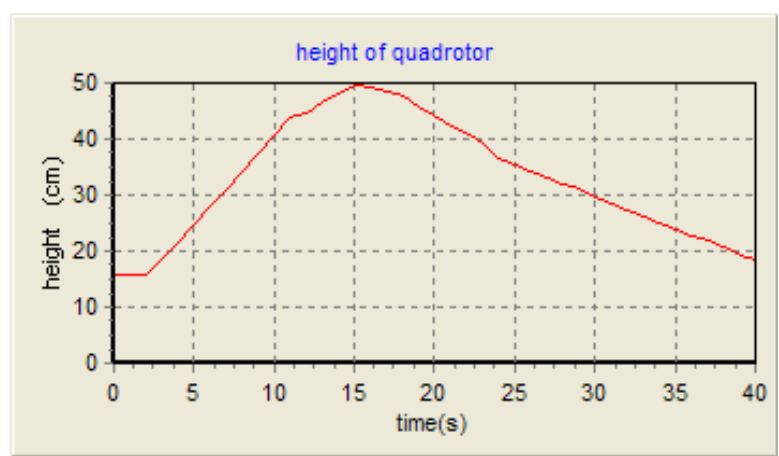

Fig. 5 The flight height of quadrotor

When the aircraft distant from the ground $50 \mathrm{~mm}$, the target object is captured by down-looking camera, and the height and attitude is adjusted by the controller to fetch objects as shown in Fig. 5.When gripper is aligned with the target, the centroid coordinates of object is located in the center 
area of the image, the image coordinates of Target as shown in Fig. 6. The steering angle of the steering gear 2 is shown in Fig.7 when the object is grasped.

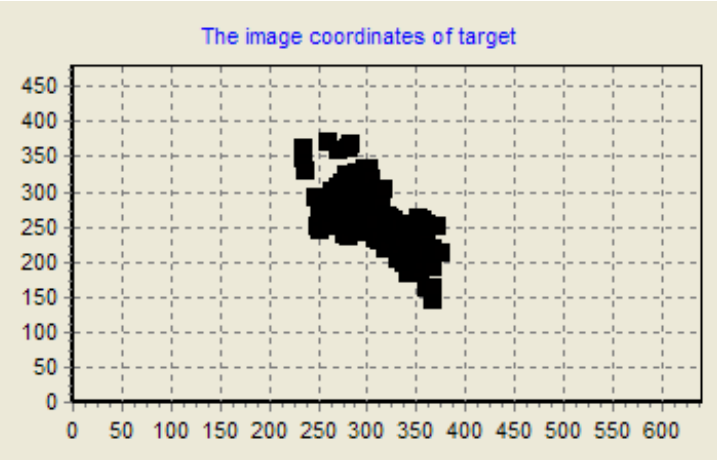

Fig. 6 the image coordinates of Target

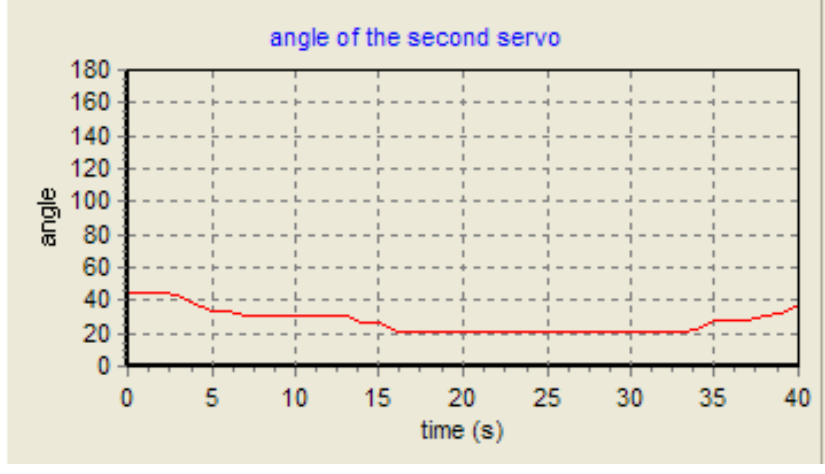

Fig. 7 the angle of the second sevro

\section{Summary}

Vision based flight grasp of four-rotor, the problem that indoor positioning of the four-rotor without the GPS positioning system is solved by down-looking camera. The impact of the positioning error caused in the process of the aircraft is reduced by end-effector camera. The method is robust to illumination change, but the change of light too large or the object is occluded, it also may cause the problem of locating missing, and the positioning is not accurate.

\section{Acknowledgments}

This work has been supported by the Innovation and Entrepreneurship Fund of Jiamusi University (xzyf2013-02) and the Natural Science Fund of Heilongjiang Province (E201254).

\section{References}

[1] Mellinger D, Lindsey Q,Shomin M, et al, Design, modeling, estimation and control for aerial grasping and manipulation[C]//2011 IEEE/RSJ International Conference on Intelligent Robots and Systems (IROS), San Francisco, 2011, pp. 2668-2673.

[2] Yazdi N, Ayazi F, Najafi K. Micromachined inertial sensors[C]//Proceedings of IEEE, 1998, pp. 1640-1659.

[3] Brett Bethke, Persistent Vision-Based Search and Track Using Multiple UAVs[D], Department of Aeronautics and Astronautics, Massachusetts Institute of Technology, 2005, pp. 22.

[4] Erdinc Altug, James P.Ostrowski, Camillo J.Taylor, Quadrotor Control Using Dual Camera Visual Feedback[C], Proceedings of the 2003 IEEE International Conference on Robotics and Automation, Taipei, Taiwan, September, 2003, pp. 14-19.

[5] Altug E, Ostrowski J, Mahony R, Control of a quadrotor helicopter using visual feedback[C]//In International Conference on Robotics and Automation, Washington, DC, 2002, pp. 72-77.

[6] Klose S, Wang J. Achtelik M, et al, Markerless, vision assisted flight control of a quadrocopter[C] //IEEE/RSJ International Conference on Intelligent Robots and Systems, Taipei, 2010, pp. 5712-5717. 